\title{
Comments on 'Emergency Management of Sigmoid Colon Volvulus in a Volvulus Belt Population and a Review of Literature'
}

\author{
Sabri Selcuk Atamanalp ${ }^{1}$ \\ Received: 15 November 2017 / Accepted: 30 January 2018 / Published online: 8 February 2018 \\ (C) Association of Surgeons of India 2018
}

Dear editor,

I read with interest the article titled 'Emergency Management of Sigmoid Colon Volvulus in a Volvulus Belt Population and a Review of Literature' by Pattanaik [1], who reported 445 patients with sigmoid volvulus (SV). Among a large number of evaluated subjects including epidemiology, diagnosis, and treatment of SV, I want to discuss some subjects including the complexity of the strategy for emergency management, the utilization of the endoscopes in non-operative treatment, and the recommendation of the emergency laparotomy in almost all cases.

I practice in Eastern Anatolia, which is an endemic area for SV. My colleagues and I have approximately 51 years of history and 1000 cases of experience with SV, which is the largest published single-center SV series in the world according to the literature in major research databases including Web of Science and PubMed [2]. In our series, non-operative detorsion was used in 723 patients with $82.4 \%$ of success, $0.7 \%$ of mortality, and $5.0 \%$ of morbidity rates. Emergency surgery was needed in 468 patients, and the mortality and morbidity rates of the emergency resection with primary anastomosis were 12.8 and $33.5 \%$, respectively. Elective surgery was applied in 111 cases with $0.0 \%$ of mortality and $11.7 \%$ of morbidity rates.

First of all, although there are still some controversial subordinate points in the treatment of SV, the principal strategy is well defined. Following an early and effective resuscitation, endoscopic detorsion is used in uncomplicated and nongangrenous cases, while emergency surgery is needed in complicated and gangrenous patients, or in patients in whom endoscopy is unsuccessful. Additionally, elective surgery is recommended in some selected patients with successful

Sabri Selcuk Atamanalp

ssa@atauni.edu.tr

1 Department of General Surgery, Faculty of Medicine, Ataturk University, 25040 Erzurum, Turkey endoscopic detorsion $[2,3]$. Secondly, although flexible endoscopy is frequently preferred by the endoscopists and better tolerated by the patients, in the non-operative treatment of SV, rigid endoscopy is also a successful procedure with a mean success rate of $70-80 \%$, in addition to its easily accessibility and inexpensiveness [2]. Finally, although the unwillingness of some patients for elective surgery is a reality, it is well known that the mortality and morbidity rates of the elective sigmoid resection and anastomosis are less than that of the emergency procedures [3]. For these reasons, I do not share the recommendation of the emergency laparotomy with primary resection as the first treatment option in almost all cases.

Although the evaluation of 366 surgically treated patients is explicit in the series presented by the author, the role of the endoscopic detorsion is not clear in the remained patients. As known, SV is endemic in India [1], where the author practices. Therefore, I hope that the author has at least a few-case experience with non-operative detorsion of SV. If he has not an additional experience on SV, particularly on endoscopic detorsion, I would at least appreciate the author's theoretical opinion on my comments.

\section{Compliance with Ethical Standards}

Conflict of Interest The author declares that he has no conflict of interest.

\section{References}

1. Pattanaik SK (2017) Emergency management of sigmoid colon volvulus in a volvulus belt population and a review of literature. Indian J Surg. https://doi.org/10.1007/s12262-017-1699-7

2. Atamanalp SS (2017) Sigmoid volvulus: the first one thousand-case single center series in the world. Eur J Trauma Emerg Surg. https:// doi.org/10.1007/s00068-017-0859-6

3. Atamanalp SS (2017) A new classification, treatment algorithm and prognosis-estimating system for sigmoid volvulus. Color Dis 19(11): 1033-1035. https://doi.org/10.1111/codi.13898 